\title{
Martina Schiavon et Laurent Rollet (dir.), Pour une histoire du Bureau des Longitudes (1795-1932)
}

Nancy, Presses universitaires de Nancy/Éditions universitaires de Lorraine, collection « Histoire des institutions scientifiques », 2017, $276 \mathrm{p}$.

Jérôme Lamy

\section{(2) OpenEdition} Journals

Édition électronique

URL : http://journals.openedition.org/artefact/2516

DOI : $10.4000 /$ artefact.2516

ISSN : 2606-9245

Éditeur :

Association Artefact. Techniques histoire et sciences humaines, Presses universitaires du Midi

Édition imprimée

Date de publication : 6 décembre 2018

Pagination : 359-362

ISBN : 978-2-8107-0595-5

ISSN : 2273-0753

Référence électronique

Jérôme Lamy, « Martina Schiavon et Laurent Rollet (dir.), Pour une histoire du Bureau des Longitudes (1795-1932) », Artefact [En ligne], 8 | 2018, mis en ligne le 21 juin 2019, consulté le 28 novembre 2020. URL : http://journals.openedition.org/artefact/2516 ; DOI : https://doi.org/10.4000/artefact.2516

Ce document a été généré automatiquement le 28 novembre 2020.

\section{c) (7) $\Theta$}

Artefact, Techniques, histoire et sciences humaines est mise à disposition selon les termes de la Licence Creative Commons Attribution - Pas d'Utilisation Commerciale - Pas de Modification 4.0 International. 


\section{Martina Schiavon et Laurent Rollet (dir.), Pour une histoire du Bureau des Longitudes (1795-1932)}

Nancy, Presses universitaires de Nancy/Éditions universitaires de Lorraine, collection « Histoire des institutions scientifiques », 2017, $276 \mathrm{p}$.

Jérôme Lamy

1 Le Bureau des Longitudes est une institution savante singulière : à proximité de l'Observatoire de Paris, sans lien avec les universités, proche des préoccupations des marins, il constitue un lieu décisif de la régulation politique des savoirs. Cet ouvrage se veut un plaidoyer en faveur d'une approche chronologique étendue et pluraliste du Bureau des Longitudes. Je donnerai ici un aperçu de certains de ses chapitres.

2 En introduction, Martina Schiavon et Laurent Rollet rappellent que l'initiative d'une structure destinée à informer l'État des connaissances nécessaires au déploiement de la navigation revient à l'abbé Grégoire, pendant la Révolution. Peu à peu, les prérogatives disciplinaires du Bureau des Longitudes s'étendent: «géodésie, mécanique céleste, cosmologie, science physique, métrologie " et même "à la fin du $19^{\mathrm{e}}$ siècle ", la "physique du globe et la géodésie dynamique»(p.12). Malgré la multiplicité des champs d'action abordés par le Bureau, Martina Schiavon et Laurent Rollet remarquent qu'il «a relativement peu intéressé les historiens» (p.13). Leur ouvrage se propose donc d'être une introduction à l'histoire d'une institution cruciale «dans l'administration de l'État ainsi que dans le contrôle et le développement de ses activités économiques » (p. 16).

3 Suzanne Débarbat, dans un chapitre récapitulatif, remet en perspective la succession des textes officiels ayant scandé l'histoire du Bureau des Longitudes. Elle rappelle qu'après l'engagement de l'abbé Grégoire, la « loi de création » (p. 25) de la nouvelle institution, en juin 1795, prévoit ses principales attributions (dont la publication à l'avance de La Connoissance des Temps). La première direction est « collégiale » (p. 29), ce qui n'est pas sans poser des problèmes d'organisation. Par la suite, la réglementation 
de 1854, est configurée aux formes du pouvoir qu'entend exercer Urbain Le Verrier. Le décret de 1874 précise les domaines scientifiques que doit couvrir le Bureau: les "diverses branches de la science astronomique et [...] leurs applications à la géophysique, à la navigation et à la physique du globe » (p. 33). Au xxe siècle, les décrets de 1970 et 1998 affinent encore la structure administrative du Bureau des Longitudes tout en conservant l'essentiel des modalités d'action.

Guy Boistel consacre un chapitre aux calculateurs « du Bureau des Longitudes et de la Connaissance des Temps ». Au XVIII ${ }^{\mathrm{e}}$ siècle, l'astronome Jérôme Lalande a profondément transformé La Connoissance des Temps en introduisant "des notices scientifiques précises et pleines de détails ». Il ajoute également « les tables des distances lunaires » (p. 49). Lalande administre un petit corps de calculateurs à son service : certains sont des membres de sa famille, d'autres sont ecclésiastiques (p. 51). La période révolutionnaire est marquée par l'institution du Bureau et par le soutien des " calculateurs du cadastre » (p. 52) aux calculs en cours. Pour la première partie du XIX siècle, Guy Boistel constate un «fonctionnement régulier de la Connaissance des Temps, avec des calculateurs dont les tâches s'inscrivent dans le temps long. Si la période Le Verrier est marquée par une déstructuration relative des capacités de calcul, la fin du XIX ${ }^{e}$ siècle voit la mise en œuvre d'un "véritable "Service des calculs" » (p. 62), entité bureaucratique régie par des modes d'accès normés. Ce chapitre échoue dans l'objectif de comparaison historique qu'il s'était fixé. Guy Boistel soutient en effet que le Bureau a, pour l'essentiel, été le lieu « d'une activité choisie et non subie » (p. 74). Il prend appui sur la longévité de certains calculateurs au sein de l'institution pour soutenir cette hypothèse. Mais la comparaison n'a de sens qu'à la condition expresse de tenir compte des contextes socio-épistémiques pour chaque époque. La bureaucratisation complète de la fin $\mathrm{du}_{\mathrm{XIX}} \mathrm{e}^{\mathrm{e}}$ siècle n'a rien à voir avec l'ébauche administrative de la fin du $\mathrm{XVIII}^{\mathrm{e}}$ siècle - et moins encore avec l'aréopage de calculateurs recrutés par Lalande dans un enchevêtrement de liens personnels.

Jean-Marie Feurtet s'intéresse, dans un chapitre particulièrement réussi, aux rapports entretenus entre l'observatoire de Marseille et le Bureau des longitudes de 1795 à 1822 . La structuration épistémique et administrative du site d'observation phocéen est déterminée par «la culture scientifique essentiellement pratique " qui y régnait à l'époque moderne (p. 92). Le rattachement "à la Marine Royale » et l'articulation à l'Académie locale fixent les grandes lignes d'un fonctionnement savant porté vers la navigation. Sous la direction de Thulis, pendant la Révolution, l'observatoire de Marseille et le Bureau des Longitudes opèrent conjointement, même si la jeune institution parisienne «peine [...] à stabiliser un personnel éloigné de Paris » (p. 100). Les liens très personnels que Thulis entretient avec l'astronome de Gotha, Franz von Zach font de l'observatoire phocéen un nœud crucial dans la carte européenne des établissements astronomiques. Von Zach « fournit instruments, conseils, contacts. Il se positionne en facilitateur, proche en cela du système de patronage paternaliste de Lalande » (p. 103). La succession de Thulis est difficile : Blanpain ne semble ni disposer du capital scientifique suffisant pour diriger l'observatoire, ni de la volonté de pérenniser l'institution. Le Bureau des Longitudes se trouve "publiquement fragilisé » lorsque Pons, qui découvrait des comètes à répétition, est rappelé par von Zach à l'observatoire de Marlia (p. 109). Au final, soutient Jean-Marie Feurtet, c'est bien la gestion de "la rareté des ressources humaines " qu'a eue à organiser le Bureau des Longitudes à Marseille. Dans un champ astronomique transformé par la révolution, 
c'est «un apprentissage pratique endogène» qui permet «la validation des compétences astronomiques » (p. 114).

Le chapitre que Richard Dunn et Rebekah Higgit consacrent aux relations entre le Bureau des Longitudes français et le Board of Longitudes britannique est éclairant sur la double dynamique entre compétition et coopération scientifique. Entre 1795 et 1828, les positions des deux institutions sont contrastées : le Bureau des Longitudes est dans sa phase émergente, le Board of Longitudes connaît une "reinvention" (p. 196) en 1818. Les auteurs soulignent que les différents blocages politiques et économiques entre les deux nations n'ont pas empêché, même avant 1815, des contacts scientifiques. L'éloge de Maskelyne que lit Delambre à l'Académie des Sciences et l'inspiration britannique de l'abbé Grégoire pour la fondation du Bureau témoignent du respect savant entretenu de part et d'autre de la Manche. Après 1815, les échanges sont plus soutenus encore: Banks reçoit ainsi des scientifiques français (notamment Arago et Biot) en 1818. La même année, le Longitude Act rénove le Board. Par la suite, les collaborations autour de la nouvelle différence de longitude mesurée entre les observatoires de Greenwich et Paris ainsi que les expériences gravitationnelles avec des pendules resserrent les liens entre les deux institutions. Finalement, si la concurrence scientifique est un moteur des rapports entre le Bureau et le Board au début du XIX siècle, la coopération et le partage des expériences constituent également des modes réguliers d'articulation entre grandes institutions savantes.

7 Frédérique Rémy explore, dans un chapitre très réussi, la façon dont le Bureau des Longitudes (et plus particulièrement François Arago) a abordé la question des «glaces polaires » (p. 243). En s'appuyant sur les textes que l'astronome français fait paraître dans l'Annuaire du Bureau des Longitudes, Frédérique Rémy met au jour les connaissances plus ou moins établies sur les pôles et leurs caractéristiques géophysiques. Arago, par ses notices, relève la synthèse des savoirs de l'époque. Il décrit ainsi "la géographie, les courants et les glaces de l'Arctique»: il soutient «l'existence d'un courant se dirigeant vers le sud au large du Groenland» (p. 249). Il évoque également une question qui nourrit de nombreux débats : le gel des rivières et des fleuves (et Arago s'intéresse beaucoup au "rayonnement nocturne» [p. 252]). L'astronome consacre également certains de ses écrits à la glace et au climat (p. 255). Il « compile les données sur le gel de différents liquides rapportées par les chroniques anciennes ou par les explorations polaires ». Il parvient à fixer « la température du pôle à $-25^{\circ} \mathrm{C} »$ (p. 256), ce qui contrevient à l'hypothèse de la «mer libre » au pôle, entourée de glaces. Arago a livré un condensé de savoirs sur l'Arctique (il ignore totalement l'Antarctique), qui signale à quel point le Bureau des Longitudes était conçu, dans cette première partie du $\mathrm{XIX}^{\mathrm{e}}$ siècle, comme un lieu d'interrogation sur des formes très variées de connaissances.

8 Finalement, cet ouvrage collectif livre des pistes de recherche particulièrement fécondes pour comprendre comment s'organise, se structure, se stabilise une institution savante dont les configurations socio-épistémiques varient dans le temps. 


\section{AUTEUR}

JÉRÔME LAMY

Université Toulouse Jean-Jaurès, CNRS - CERTOP 